\title{
Genomic imbalances during transformation from follicular lymphoma to diffuse large B-cell lymphoma
}

\author{
Mattias Berglund ${ }^{1,2}$, Gunilla Enblad ${ }^{2}$, Ulf Thunberg ${ }^{2}$, Rose-Marie Amini ${ }^{3}$, Christer
} Sundström ${ }^{2}$, Göran Roos ${ }^{4}$, Martin Erlanson ${ }^{5}$, Richard Rosenquist' ${ }^{3}$, Catharina Larsson ${ }^{1}$ and Svetlana Lagercrantz ${ }^{1}$

\author{
${ }^{1}$ Department of Molecular Medicine and Surgery, Karolinska University Hospital - Solna, Stockholm, \\ Sweden; ${ }^{2}$ Department of Oncology, Radiology, and Clinical Immunology, Section of Oncology, Uppsala \\ University, Uppsala, Sweden; ${ }^{3}$ Rudbeck Laboratory, Department of Genetics and Pathology, Uppsala \\ University, Uppsala, Sweden; ${ }^{4}$ Department of Medical Biosciences, Section of Pathology, Umea University, \\ Umea ${ }^{\circ}$ Sweden and ${ }^{5}$ Department of Radiation Sciences, Section of Oncology, Umea University, Umea, \\ Sweden
}

\begin{abstract}
Follicular lymphoma is commonly transformed to a more aggressive diffuse large B-cell lymphoma (DLBCL). In order to molecularely characterize this histiological and clinical transformation, comparative genomic hybridization was applied on $\mathbf{2 3}$ follicular lymphoma and 35 transformed DLBCL tumors from a total of 30 patients. The results were also compared with our published findings in de novo DLBCL. Copy number changes were detected in $70 \%$ of follicular lymphoma and in $97 \%$ of transformed DLBCL. In follicular lymphoma, the most common alterations were $+18 q 21(33 \%),+$ Xq25-26 (28\%), +1q31-32 (23\%), and $-17 p(23 \%)$, whereas transformed DLBCL most frequently exhibited + Xq25-26 (36\%), +12q15 (29\%), + 7pter-q22 (25\%), +8q21 (21\%), and $-6 \mathrm{q} 16-21(25 \%)$. Transformed DLBCL showed significantly more alterations as compared to follicular lymphoma $(P=0.0001)$, and the alterations $-6 q 16-21$ and $+7 p$ ter-q22 were only found in transformed DLBCL but not in follicular lymphoma $(P=0.02)$. Alterations involving $+13 q 22$ were significantly less frequent, whereas -4q13-21 was more common in transformed as compared to de novo DLBCL $(P=0.01$ and $P=0.02$, respectively). Clinical progression from follicular lymphoma to transformed DLBCL is on the genetic level associated with acquirement of increasing number of genomic copy number changes, with non-random involvement of specific target regions. The findings support diverse genetic background between transformed and de novo DLBCL.

Modern Pathology (2007) 20, 63-75. doi:10.1038/modpathol.3800708; published online 29 September 2006
\end{abstract}

Keywords: lymphoma; transformation; comparative genomic hybridization

Follicular lymphoma is one of the most common types of lymphoma and is recognized as a distinct entity both clinically and genetically. The tumors are derived from germinal center B cells, and are strongly associated with a characteristic translocation $\mathrm{t}(14 ; 18)(\mathrm{q} 32 ; \mathrm{q} 21)$ found in up to $80 \%$ of the cases. ${ }^{1}$ Following this chromosomal rearrangement, $B C L-2$ expression is increased, which in turn is believed to prolong cell survival and facilitate accumulation of genetic changes involved in the

Correspondence: Dr M Berglund, PhD, Department of Molecular Medicine and Surgery, Karolinska University Hospital - Solna, CMM L8:01, Stockholm SE-171 76, Sweden.

E-mail: Mattias.Berglund@genpat.uu.se

Received 14 June 2006; revised and accepted 23 August 2006; published online 29 September 2006 malignant transformation. ${ }^{2-4}$ Patients with follicular lymphoma normally show an initial indolent clinical course, but eventually almost all succumb to the disease. This is partly related to the frequent transformation of follicular lymphoma to aggressive diffuse large B-cell lymphoma (DLBCL). ${ }^{5}$ Transformation of DLBCL is diagnosed in up to $10-60 \%$ of follicular lymphoma cases and is associated with an accelerated clinical course and shorter survival. ${ }^{5}$ Thus, follicular lymphoma represents a clinical dilemma as its natural course cannot be predicted, and in addition the prognosis after transformation to DLBCL is poor in spite of treatment with combination chemotherapy.

DLBCL can be classified as de novo, that is, without a previous history of an indolent lymphoma, or as secondary transformed DLBCL emanating 
from an indolent lymphoma most commonly follicular lymphoma. The distinction between transformed and de novo DLBCL is based on the morphological demonstration of a low malignancy grade lymphoma such as follicular lymphoma either before or simultaneous to the DLBCL. However, it is not known whether some apparently de novo DLBCL could in fact represent transformed cases where the component of low-grade malignancy is no longer detectable. The demonstration of a $t(14 ; 18)$ rearrangement in a significant proportion of de novo DLBCL gives some support for this hypothesis. ${ }^{4}$

Several genetic events have previously been proposed to be associated with transformation of follicular lymphoma to DLBCL, including TP53 mutations, ${ }^{6}$ gene activation of $M Y C,{ }^{7}$ deregulation of $R E L,{ }^{8}$ somatic mutations of the translocated $B C L-2$ gene in $18 \mathrm{q} 21,{ }^{9}$ as well as deletions at $9 \mathrm{p} 21^{10}$ involving $p 15^{I N K 4 b}$ and $p 16^{I N K 4 a}$. Other approaches include, for example, screenings on the genomic and RNA expression levels using comparative genomic hybridization (CGH), and different micro-arraybased techniques. ${ }^{1-14}$ Global gene expression profiling have shown highly similar expression signatures in ancestral follicular lymphoma and transformed DLBCL, whereas de novo DLBCL clustered separately, suggesting etiological differences between transformed and de novo DLBCL. ${ }^{15}$ In studies of genomic copy number alterations, gains involving $+1 \mathrm{q},+3 \mathrm{q},+7,+12,+18 \mathrm{q} 21$, and $+\mathrm{Xq}$ as well as losses in $-4 q,-6 q$, and $-17 p$ have been associated with transformation from follicular lymphoma to DLBCL. ${ }^{12,13,16}$

In this study, we comprehensively characterize genomic copy number changes associated with the transformation from follicular lymphoma to DLBCL as well as tumor progression using CGH analysis. The clonality was in selected cases determined by single-stranded conformation polymorphism (SSCP) and sequencing of the $I G H$ gene. Furthermore the results for transformed DLBCL were compared to our previously published findings in de novo DLBCL. ${ }^{17}$

\section{Materials and methods}

\section{Patients and Tumor Specimens}

Thirty patients (13 men and 17 women) diagnosed between 1970 and 1997 with a follicular lymphoma, and a later transformed DLBCL, were included in the study (Table 1). The patients were identified from the files of the Departments of Pathology at the Uppsala University Hospital and the Umea University Hospital, Sweden. A total of 58 frozen tumor biopsies were available from the 30 patients, and were all collected with approval from an ethical committee. All tumors were histopathologically reevaluated by two of the authors (RA and GR), according to the WHO classification. ${ }^{18}$ This confirmed the initial diagnoses of follicular lymphoma and DLBCL in all cases. Tumors were diagnosed as transformed DLBCL based on their previous history of follicular lymphoma. Four patients had a discordant lymphoma with simultaneous diagnosis of follicular lymphoma and DLBCL (cases 3, 5, 14, and 18), and in these cases the CGH analysis was performed on the transformed DLBCL. The other 26 patients were first diagnosed with follicular lymphoma, and then presented a transformed DLBCL after a median time of 60 months (range 0.5-247 months). From 16 of these 26 patients, samples from both follicular lymphoma and transformed DLBCL were available, however not always from the first diagnosis of follicular lymphoma and DLBCL, respectively (Tables 1 and 2). From case 30, four samples of follicular lymphoma and two transformed DLBCL were analyzed. From cases 1 and 2, only follicular lymphoma material was studied, whereas from cases 3-14 material was only available from the transformed DLBCL. For eight cases $(15,17,20,21,22,28,29$, and 30), paraffin blocks for immunohistochemistry were available from matched tumors, that is, from the follicular lymphoma as well as DLBCL counterpart. A total of 16 paired cases were available for CGH (including the eight cases available for immunohistochemistry; see Table 2). For seven of the cases, the CGH have been previously published (tumor no. $3,4,5,10$, 12:1, 12:2; 13:1, 13:2, 14:1, 14:2, and 14:3 in Table 2; corresponding to no. 25, 21, 23, 13, 33D, 33R, 32D, 32R, 34D, 34R1, and 34R2). ${ }^{17}$ After re-evaluating the original CGH data, $-16 \mathrm{p}$ was added to the findings in tumors 14:2 and 14:3 (34R1 and R2).

\section{CGH Analysis}

High molecular weight DNA was prepared from frozen tumor material using QIAamp DNA Mini Kit (Qiagen, Valencia, CA, USA) including proteinase K treatment. CGH was performed using methods described previously. ${ }^{17}$ Briefly, tumor DNA was labeled with FITC and the reference DNA with Texas-Red (Vysis Inc., Downers Growe, IL, USA), mixed with Cot-I DNA, and hybridized together onto denatured metaphase spreads from normal lymphocytes (Vysis Inc.). The slides were counterstained with 4,6-diamidino-2-phenylindole (DAPI, Vysis, Inc). Ten to twelve three-color digital images were captured from each hybridized slide using an Axioplan 2 epifluorescence microscope (Carl Zeiss Jena GmbH, Jena, Germany) equipped with Sensys cooled charge-coupled device camera (Photometrics) and analyzed with the isis/CGH software (Metasystems, Altlussheim, Germany). For each hybridization, at least 10 ratio profiles were averaged for each chromosome to reduce noise. Green to red ratios below 0.8 were considered as losses, above 1.2 as gains, and above 1.5 as high-level amplifications. Heterochromatic regions in the centromeric and paracentromeric parts of the chromo- 
Table 1 Clinical characteristics of the 30 patients with transformation from follicular lymphoma to diffuse large B-cell lymphoma

\begin{tabular}{|c|c|c|c|c|c|c|c|c|}
\hline \multirow[t]{2}{*}{ Case no. } & \multirow{2}{*}{$\begin{array}{c}\text { Age at } \\
\text { diagnosis } \\
\text { (yrs) }\end{array}$} & \multirow[t]{2}{*}{ Sex } & \multirow[t]{2}{*}{ Date of diagnosis } & \multirow{2}{*}{$\begin{array}{l}\text { Treatment of } \\
\text { follicular } \\
\text { lymphoma }\end{array}$} & \multirow{2}{*}{$\begin{array}{c}\text { Time to } \\
\text { transformation } \\
\text { (m) }\end{array}$} & \multirow{2}{*}{$\begin{array}{l}\text { Treatment } \\
\text { of diffuse } \\
\text { large B-cell } \\
\text { lymphoma }\end{array}$} & \multicolumn{2}{|c|}{ Follow-up } \\
\hline & & & & & & & Time (m) & Outcome \\
\hline 1 & 73 & $\mathrm{M}$ & August 1987 & NA & 0.5 & NA & NA & DoL \\
\hline 2 & 59 & $\mathrm{~F}$ & February 1987 & $\begin{array}{l}\text { CHOP, } \\
\text { chlorambucil }\end{array}$ & 60 & $\mathrm{CT}$ & 26 & DoL \\
\hline 3 & 50 & $\mathrm{~F}$ & September 1994 & - & 0 & CT & 97 & CCR \\
\hline 4 & 62 & $\mathrm{M}$ & September 1982 & $\begin{array}{l}\text { RT, } \\
\text { chlorambucil }\end{array}$ & 107 & CT & 18 & DoL \\
\hline 5 & 48 & $\mathrm{M}$ & August 1989 & - & 0 & MACOP-B, RT & 130 & DoL \\
\hline 6 & 50 & $\mathrm{~F}$ & February 1981 & $\begin{array}{l}\text { WW, RT, alfa- } \\
\text { interferon }\end{array}$ & 88 & CHOP & 7 & DoL \\
\hline 7 & 51 & $\mathrm{~F}$ & October 1987 & $\begin{array}{l}\text { RT, } \\
\text { chlorambucil, } \\
\text { Fludara }\end{array}$ & 110 & CNOP & 5 & DoL \\
\hline 8 & 49 & $\mathrm{M}$ & November 1997 & $\begin{array}{l}\text { chorambucil, } \\
\text { RT }\end{array}$ & 15 & $\begin{array}{l}\text { CHOP, RT, } \\
\text { ABMT }\end{array}$ & 25 & DoL \\
\hline 9 & NA & $\mathrm{F}$ & NA & NA & NA & NA & NA & NA \\
\hline 10 & 51 & $\mathrm{M}$ & February 1986 & RT & 44 & CHOP & 129 & CCR \\
\hline 11 & 57 & $\mathrm{M}$ & May 1988 & CHOP & 127 & CT & 57 & DoL \\
\hline 12 & 58 & $\mathrm{~F}$ & December 1981 & $\begin{array}{l}\text { Chlorambucil, } \\
\text { RT }\end{array}$ & 123 & CT & 98 & DoL \\
\hline 13 & 61 & $\mathrm{M}$ & July 1983 & $\begin{array}{l}\text { WW, RT, } \\
\text { chlorambucil }\end{array}$ & 61 & CHOP & 1 & DoL \\
\hline 14 & 62 & $\mathrm{~F}$ & November 1985 & - & 0 & CT & 84 & DoL \\
\hline 15 & 49 & $\mathrm{M}$ & December 1989 & $\begin{array}{l}\text { Chlorambucil, } \\
\text { CT, ABMT }\end{array}$ & 140 & CT & NA & DoL \\
\hline 16 & 37 & $\mathrm{M}$ & June 1982 & RT & 93 & CHOP, ABMT & 26 & DoL \\
\hline 17 & 61 & M & September 1990 & RT & 76 & CHOP & 3 & DoL \\
\hline 18 & 33 & $\mathrm{~F}$ & November 1992 & - & 0 & CHOP, АBMT & 126 & CCR \\
\hline 19 & 71 & $\mathrm{M}$ & January 1988 & $\begin{array}{l}\text { WW, } \\
\text { chlorambucil }\end{array}$ & 90 & $\mathrm{CHOP}$ & 7 & DoL \\
\hline 20 & 69 & $\mathrm{~F}$ & July 1994 & WW & 12 & CHOP & 12 & DoL \\
\hline 21 & 48 & $\mathrm{~F}$ & February 1985 & Chlorambucil & 60 & CHOP & 21 & DoL \\
\hline 22 & 25 & $\mathrm{~F}$ & October 1970 & $\begin{array}{l}\text { RT, } \\
\text { chlorambucil }\end{array}$ & 247 & CHOP & 1 & DoL \\
\hline 23 & 50 & $\mathrm{~F}$ & February 1991 & $\begin{array}{l}\text { chlorambucil, } \\
\text { CHOP }\end{array}$ & 48 & MIME & 38 & DoL \\
\hline 24 & 57 & M & February 1994 & Chlorambucil & 13 & CT, ABMT & 123 & CCR \\
\hline 25 & 60 & $\mathrm{M}$ & October 1997 & Chlorambucil & 14 & CHOP & 78 & CCR \\
\hline 26 & 71 & $\mathrm{~F}$ & May 1984 & RT & 9 & COP & 4 & DoL \\
\hline 27 & 60 & $\mathrm{~F}$ & January 1987 & Splenectomy & 3 & CHOP & 132 & DoL \\
\hline 28 & 51 & $\mathrm{~F}$ & June 1992 & $\begin{array}{l}\text { CHOP, } \\
\text { chlorambucil }\end{array}$ & 58 & CT & 61 & DoL \\
\hline 29 & 39 & $\mathrm{~F}$ & December 1992 & $\begin{array}{l}\text { WW, } \\
\text { chlorambucil, } \\
\text { CHOP }\end{array}$ & 96 & MIME & 40 & DoL \\
\hline 30 & 46 & $\mathrm{~F}$ & August 1987 & $\begin{array}{l}\text { WW, } \\
\text { chlorambucil }\end{array}$ & 148 & CT & 73 & DoL \\
\hline
\end{tabular}

ABMT, high dose therapy with autologous stem cell rescue; CCR, continous complete remission; CHOP, cyclofosfamide, vincrisine, adriamycin, and prednisolone; CNOP, cyclofosfamide, vincristine, mitoxantrone, and prednisolone; COP, cyclofosfamide, vincristine, and prednisolone; CT, different combination of chemotherapy; DoL, dead of lymphoma; m, months; MACOP-B, methotrexate, adriamycin, cyclofosfamide, vincrisitne, bleomycin, and prednisolone; MIME, methyl-gag, ifosfamide, methotrexate, and etoposide; NA, not available; RT, radiotherapy; WW, wait and watch; yrs, years.

somes, as well as the short arms of the acrocentric chromosomes, and the telomeric regions were not included in the evaluation. As an internal control, 10 of the tumor samples were independently hybridized and analyzed on at least two occasions, giving identical results in all cases. DNA samples from aberrant cancer cell lines and normal individuals were similarly analyzed as positive and negative controls, respectively.

\section{Immunohistochemistry}

Formalin-fixed paraffin sections $4-\mu \mathrm{m}$ thick were deparaffinized and rehydrated. Mouse monoclonal bcl-2 (diluted 1/10, clone 124; Dako, Glostrup, Denmark), antibody stainings were performed in a Ventana benchmark machine (Ventana medical systems, Tucson, USA). Buffer CC2 (Ventana) was used for antigen retrieval. Primary antibodies were 
Table 2 Copy number aberrations detected by CGH in FL and transformed DLBCL

\begin{tabular}{|c|c|c|c|c|}
\hline $\begin{array}{l}\text { Tumor } \\
\text { no. }\end{array}$ & Diagnosis & Bcl-2 (\%) & Number & $\begin{array}{l}\text { CGH alterations } \\
\text { Regions involved }\end{array}$ \\
\hline 1 & $\mathrm{FL}^{\mathrm{III}}$ & NA & 0 & - \\
\hline 2 & $\mathrm{FL}^{\mathrm{I}}$ & 75 & 0 & - \\
\hline 3 & DLBCL & NA & 0 & - \\
\hline 4 & DLBCL & NA & 2 & -8p22-pter,-9p23-pter \\
\hline 5 & DLBCL & NA & 3 & + Xp11.3-q26,-8p22-pter, $-9 \mathrm{p} 22$-pter \\
\hline 6 & DLBCL & 0 & 3 & $+4,+6$ pter-q14,+12pter-q22 \\
\hline 7 & DLBCL & NA & 3 & $-5 p,+7,-11 q 22-25$ \\
\hline 8 & DLBCL & 0 & 4 & $-4 q 10-22,-6 q 14-21,+12$ pter-q22,+18q \\
\hline 9 & DLBCL & NA & 6 & -Xpter-q13,+3q26-qter,-4p16,-6q16-qter,+11q13-22,-15 \\
\hline 10 & DLBCL & NA & 7 & +1q31-qter,-2p23-pter,-4q13-21,-8p12-pter,+11q22-23,+12p11-q21,-18q22-qter \\
\hline 11 & DLBCL & 100 & 9 & $+2 \mathrm{p} 14-16,+2 \mathrm{q} 21-32,-3 \mathrm{p} 14-21,-3 \mathrm{q} 13-22,+6 \mathrm{p},+7$ pter-q22,+8,-18q22-23,+21 \\
\hline $12: 1$ & DLBCL & NA & 5 & $-X,+1 q,-4 p,+6 p,-6 q 23-q t e r$ \\
\hline $12: 2$ & DLBCL & NA & 4 & $-X,+1 q,+6 p,-6 q 23-q$ ter \\
\hline $13: 1$ & DLBCL & 80 & 11 & $\begin{array}{l}-1 p,-2 q 33-q t e r,-4 p t e r-q 21,-6 p 21-q 21,+7,+10 p 12-p t e r,-10 q 23-q t e r,-11 q,+12 p 12- \\
q 21,+14 q 12-21,+21 q\end{array}$ \\
\hline $13: 2$ & DLBCL & 80 & 10 & $+\mathrm{X},-1 \mathrm{p} 32-$-pter, $+7,+10 \mathrm{p} 12-$-pter,-10q23-qter,-11q,+12p12-q21,+14q12-21,+21q, $-22 \mathrm{q}$ \\
\hline $14: 1$ & DLBCL & NA & 8 & $+X,-1 p 21-p t e r,-6 q,+7,-10 q 22-23,-15 q,-16 p,-17 p$ \\
\hline $14: 2$ & DLBCL & NA & 12 & $\begin{array}{l}+\mathrm{X},-1 \mathrm{p} 21-\text { pter, }-3 \mathrm{q} 13.1-21,-6 \mathrm{q},+7,-10 \mathrm{q} 22-23,-15 \mathrm{q},-16 \mathrm{p},-17,-18 \mathrm{pter}- \\
\mathrm{q} 21,+19 \mathrm{p},+21 \mathrm{q}\end{array}$ \\
\hline $14: 3$ & DLBCL & NA & 12 & $\begin{array}{l}+X,-1 p 21-p t e r,-3 q 13.1-21,-6 q,+7,-10 q 22-23,-15 q,-16 p,-17,-18 p t e r- \\
q 21,+19 p,+21 q\end{array}$ \\
\hline $15: 1$ & $\mathrm{FL}^{\mathrm{I}}$ & 80 & 2 & $+\mathrm{X},-9 \mathrm{p} 12-21$ \\
\hline $15: 2$ & DLBCL & 0 & 5 & $+4 q 31$-qter, $-9 q 22,+11 q 14-22,+13 q 12-22,+\mathbf{1 8 q} 12-22$ \\
\hline $16: 1$ & $\mathrm{FL}^{\mathrm{NA}}$ & 50 & 0 & - \\
\hline $16: 2$ & DLBCL & NA & 8 & $+X q,-1 p 32-p t e r,-4 q 21-q t e r,-8 p,+8 q 21-q t e r,+9 q 22-q t e r,-15,-17 p$ \\
\hline $17: 1$ & FL $^{\text {II }}$ & 50 & 2 & $+\mathrm{X},+5 \mathrm{p}$ \\
\hline $17: 2$ & DLBCL & 0 & 4 & $+X,+5 p,+12 q 10-21,-16 q 21-23$ \\
\hline $18: 1$ & $\mathrm{FL}^{\mathrm{I}}$ & NA & 0 & - 1 - 1 \\
\hline $18: 2$ & DLBCL & NA & 9 & $+2 \mathrm{q} 22-33,+4 \mathrm{q},-6 \mathrm{p} 21,+6 \mathrm{q} 10-21,+8 \mathrm{q} 12-24,+9 \mathrm{p},-11 \mathrm{q} 12-13,+12 \mathrm{q} 15-22,+12 \mathrm{p} 12$ \\
\hline 19:1 & FL $^{\mathrm{II}}$ & NA & 3 & $+12 q 11-21,-17 p,+18 p t e r-q 21$ \\
\hline $19: 2$ & DLBCL & 100 & 4 & $-6 q 15-23,+11,-17 p,+18 p t e r-q 21$ \\
\hline $20: 1$ & $\mathrm{FL}^{\mathrm{I}}$ & 50 & 3 & $+12 q 23-24,+17,+19$ \\
\hline $20: 2$ & DLBCL & 50 & 3 & $+12 q 23-24,+17,+19$ \\
\hline $21: 1$ & $\mathrm{FL}^{\mathrm{I}}$ & 50 & 1 & $+1 \mathrm{q}$ \\
\hline $21: 2$ & DLBCL & 50 & 2 & $+1 \mathrm{q},+7$ \\
\hline $22: 1$ & $\mathrm{FL}^{\mathrm{I}}$ & 90 & 2 & $+12 q 13-15,+18 q 21$ \\
\hline $22: 2$ & DLBCL & 75 & 4 & $-8 p,+12 q 13-15,+18 q 21,-19 p t e r-13.2$ \\
\hline $23: 1$ & $\mathrm{FL}^{\mathrm{I}}$ & 100 & 1 & $+18 p$ \\
\hline $23: 2$ & DLBCL & NA & 1 & $-4 q^{2}$ \\
\hline $24: 1$ & $\mathrm{FL}^{\mathrm{III}}$ & NA & 3 & $+1 q 23-32,-6 q 24-26,+18$ \\
\hline $24: 2$ & DLBCL & NA & 2 & +Xq21-qter,+11p \\
\hline $25: 1$ & $\mathrm{FL}^{\mathrm{I}}$ & NA & 4 & $+1 q,+11,+18 q,+21 q$ \\
\hline $25: 2$ & DLBCL & 80 & 4 & $+X,+11,+\mathbf{1 8 q},+21 q$ \\
\hline $26: 1$ & $\mathrm{FL}^{\mathrm{I}}$ & NA & 6 & $-9 p,+9 q 22-q$ ter, $-12 p,-13,-17 p,+18$ \\
\hline $26: 2$ & DLBCL & 75 & 7 & $-1 p 21-32,-9 p,+9 q 22-q t e r,-12 p,-13,-17 p,-19 p$ \\
\hline $27: 1$ & $\mathrm{FL}^{\mathrm{I}}$ & NA & 0 & - \\
\hline $27: 2$ & DLBCL & 25 & 2 & $+8 p,+8 q 21.3-q$ ter \\
\hline $27: 3$ & DLBCL & NA & 5 & $+\mathrm{X},+9$ pter-31,+15,+18pter-21,+19q13.2-qter \\
\hline $28: 1$ & $\mathrm{FL}^{\mathrm{I}}$ & 90 & 3 & $+X,-6 q 13-15 ; 22-23$ \\
\hline $28: 2$ & DLBCL & 100 & 5 & + Xq25-qter,+2p14-16,-6q13-15; 22-23,+7 \\
\hline 28:3 & DLBCL & 80 & 5 & $+X,+2 p 14-16,-6 q 13-15 ; 22-23,+7$ \\
\hline 29:1 & $\mathrm{FL}^{\mathrm{I}}$ & 50 & 6 & $+4,-12 q 23-24,+13 q 21-31,-16 p,-17,+18$ \\
\hline $29: 2$ & $\mathrm{FL}^{\mathrm{I}}$ & 75 & 5 & $+4,-12 q 23-24,+13 q 21-31,-16 p,+18$ \\
\hline $29: 3$ & $\mathrm{FL}^{\mathrm{II}}$ & 0 & 5 & $+2 p 13-21,+11 p,+15 q 11-14,-15 q 15-q t e r,+19$ \\
\hline $29: 4$ & DLBCL & 25 & 12 & $\begin{array}{l}-X+2 p 13-21,+3 q 25-q t e r,-4 q 32-33,-6 q 14-25,+7 p t e r-q 31,+8 q 10-22,+10 p,-13 q 22- \\
\text { qter,+15q11-14,-15q15-qter,-17p, }\end{array}$ \\
\hline $30: 1$ & $\mathrm{FL}^{\mathrm{II}}$ & NA & 0 & - \\
\hline $30: 2$ & $\mathrm{FL}^{\mathrm{II}}$ & NA & 0 & - \\
\hline $30: 3$ & $\mathrm{FL}^{\mathrm{II}}$ & 80 & 2 & $+\mathrm{X},+1 \mathrm{q}$ \\
\hline $30: 4$ & $\mathrm{FL}^{\mathrm{I}}$ & NA & 2 & $+\mathrm{X},+1 \mathrm{q}$ \\
\hline $30: 5$ & DLBCL & 50 & 4 & $+X,+1 q,-6 q,+17 q$ \\
\hline $30: 6$ & DLBCL & NA & 5 & $+X,+1 q,-6 q,+12,+17 q$ \\
\hline
\end{tabular}

I-III, grading concerning FL; DLBCL, diffuse large B-cell lymphoma; FL, follicular lymphoma; NA, not available. Bold style indicates high-level amplification. 
incubated for $30 \mathrm{~min}$ and the $\mathrm{DAB}$ detection kit (Ventana) was used for the staining. Normal goat and mouse sera (Dakopatts) were used as negative controls and sections of reactive tonsil as positive controls. The proportion of positively stained tumor cells was visually estimated by a pathologist (RA). For each case, the hot spot with the highest percentage of tumor cells was used for analysis. Cases were considered as positive for bcl-2 if $50 \%$ or more of the tumor cells were positively stained.

\section{Clonality Analysis}

In order to evaluate the clonality in cases showing different CGH profiles, that is, cases 27 and 29, IGH rearrangements were used as a marker for clonality. Two cases, 20 and 22, in which the CGH profiles were similar between the follicular lymphoma and DLBCL tumors were also analyzed. $V_{\mathrm{H}}$ gene familyspecific PCR amplification was performed using specific $V_{\mathrm{H}} / J_{\mathrm{H}}$ primers as described previously. ${ }^{19,20}$ To distinguish monoclonal PCR-products from polyclonal, a SSCP analysis was performed using polyacrylamide gel or GenePhor system electrophoresis (Pharmacia LKB Biotechnology, Uppsala, Sweden) as reported previously. ${ }^{21}$ For cases 20, 22, and 29, clonal PCR products amplified from tumors were direct sequenced using the BigDye Terminator Cycle Sequencing Reaction Kit (Perkin-Elmer, ABI, FosterCity, CA, USA).

\section{Statistical Analysis}

Differences in proportions of CGH alterations were analyzed with Fisher's exact test. The distributions of total number and individual CGH alterations between follicular lymphoma and transformed DLBCL were analyzed with the Mann-Whitney $U$ test. Similar analyses were performed to compare the CGH alterations reported here in transformed DLBCL with published CGH data for de novo DLBCL. ${ }^{17}$ All statistical analyses were performed using the Statistica 6.1 software (Stat Soft Inc., Tulsa, USA). $P$-values less than 0.05 were considered as statistically significant.

\section{Results}

\section{Bcl-2 Expression by Immunohistochemistry}

Immunohistochemistry was performed on a total of 31 tumors (Table 2), of which 13 were follicular lymphoma and 18 DLBCL. In the remaining cases, no tumor material was available for immunohistochemistry. Among the analyzed follicular lymphomas, 12 tumors (92\%) showed positive bcl-2 staining, whereas $14(78 \%)$ of the DLBCL tumors were positively stained.
CGH Alterations in Follicular Lymphoma and Transformed DLBCL

In this study, genomic copy number alterations were characterized in a panel of 23 follicular lymphomas and 35 transformed DLBCLs from 30 patients with histopathologically confirmed transformation of follicular lymphoma to DLBCL. The clinical characteristics are detailed in Table 1, and the findings are shown in Tables 2-6 as well as Figures 1 and 2 . In the entire material of 58 tumors, gains of chromosomal regions were more frequently encountered than losses (Figure 1a and b). Gains most commonly involved + Xq25-26, + 1q31-32, + 7pter-q22, $+8 \mathrm{q} 21.3-22, \quad+11 \mathrm{q} 22, \quad+12 \mathrm{q} 15, \quad+18 \mathrm{q} 21$, and $+21 \mathrm{q}$, whereas losses most frequently encompassed $-4 q 13-21,-6 q 16-21,-8 p 22-p t e r$, and $-17 p$. High-level amplifications were noted as $++2 \mathrm{p} 14-16$, ++7 pter-q22, $++12 \mathrm{p} 11-\mathrm{q} 21$, and $++18 \mathrm{q}$.

Several differences in CGH alterations were noted between the groups of follicular lymphoma and transformed DLBCL studied (Table 3). In Table 3, the most commonly occurring alterations, that is, found in four cases or more, are listed. Significantly higher total numbers of CGH alterations were observed in transformed DLBCL as compared to follicular lymphoma (mean 5.4 and 2.2, respectively, $P=0.0001)$. In follicular lymphoma, the most common gains were $+18 q 21(33 \%)$, + Xq25-26 $(28 \%)$, $+1 q 31-32(23 \%)$, and $+12 q 15(11 \%)$, whereas the most common loss was $-17 \mathrm{p}(23 \%)$. In transformed DLBCL, + Xq25-26 (36\%), + 12q15 (29\%), + 7pterq22 $(25 \%)$, and $+18 \mathrm{q} 21(21 \%)$ were the most common gains, whereas losses involved -6q16-21 (25\%), -8p22-pter (18\%), and $-17 p(18 \%)$. Two of the common changes $-17 p$ and $+12 q 15$ never cooccurred in the same tumor or patient. Moreover, -6q16-21 and + 7pter-q22 were frequently detected in transformed DLBCL but never in follicular lymphoma (Figure 1; $P=0.02$ and 0.02 , respectively).

\section{Genetic Progression Associated with Transformation of Follicular Lymphoma to DLBCL}

Two approaches were taken to determine the order of chromosomal imbalances in the genetic progression and its possible association with transition from follicular lymphoma to transformed DLBCL. Firstly, the tumors were organized with an ascending number of CGH aberrations. Imbalances were considered as early events if found in tumors with low total number of changes, and as late events if they were only present in cases with high total number of changes. In follicular lymphoma, gains involving +1q31-32, + Xq25-26, + 18q21, and +12 q15 were all detected as early events (Table 4 ). Partly in contrast, $-4 q 13-21$, + Xq25-26, -8p22pter, -9p23-pter, + 7pter-q22, and +1q31-32 appeared relatively early in transformed DLBCL, 
Table 3 Most common CGH alterations in transformed DLBCL as compared to preceding FL as well as de novo DLBCL

\begin{tabular}{|c|c|c|c|c|c|}
\hline $\begin{array}{l}\text { Data from } \\
\text { alteration }\end{array}$ & $\begin{array}{l}\text { This study, } \\
F L(\mathrm{n}=18)\end{array}$ & $\mathrm{P}$-value & $\begin{array}{c}\text { This study, } \\
\text { transformed DLBCL } \\
(\mathrm{n}=28)\end{array}$ & $\mathrm{P}$-value & $\begin{array}{l}\text { Berglund et } \mathrm{al}^{17} \text { de } \\
\text { novo } D L B C L(\mathrm{n}=33)\end{array}$ \\
\hline$+\mathrm{Xq} 25-26$ & $5 / 18(28 \%)$ & NS (0.6) & $10 / 28(36 \%)$ & NS (0.4) & $15 / 33(45 \%)$ \\
\hline -1q34-qter & $0 / 18(0 \%)$ & NS (0.2) & $3 / 28(11 \%)$ & NS $(0.2)$ & $8 / 33(24 \%)$ \\
\hline$+1 q 31-32$ & $4 / 18(23 \%)$ & NS (0.5) & $4 / 28(14 \%)$ & NS (0.07) & $2 / 33(6 \%)$ \\
\hline$+3 q 24-25$ & $0 / 18(0 \%)$ & NS (0.4) & $1 / 28(4 \%)$ & 0.04 & $7 / 33(21 \%)$ \\
\hline$-4 q 13-21$ & $1 / 18(6 \%)$ & NS (0.4) & $4 / 28(14 \%)$ & 0.02 & $0 / 33(0 \%)$ \\
\hline$-6 q 16-21$ & $0 / 18(0 \%)$ & 0.02 & $7 / 28(25 \%)$ & NS (0.9) & $8 / 33(24 \%)$ \\
\hline +7pter-q22 & $0 / 18(0 \%)$ & 0.02 & $7 / 28(25 \%)$ & NS (0.3) & $5 / 33(15 \%)$ \\
\hline -8p22-pter & $1 / 18(6 \%)$ & NS (0.2) & $5 / 28(18 \%)$ & NS (0.5) & $8 / 33(24 \%)$ \\
\hline$+8 q 21.3-22$ & $0 / 18(0 \%)$ & NS (0.06) & $5 / 28(18 \%)$ & NS (0.5) & $4 / 33(12 \%)$ \\
\hline -9p23-pter & $1 / 18(6 \%)$ & NS $(0.5)$ & $3 / 28(11 \%)$ & NS (0.9) & $4 / 33(12 \%)$ \\
\hline$+11 q 22$ & $1 / 18(6 \%)$ & NS $(0.2)$ & $5 / 28(18 \%)$ & NS (0.8) & $5 / 33(15 \%)$ \\
\hline$+12 q 15$ & $2 / 18(11 \%)$ & NS (0.2) & $8 / 28(29 \%)$ & NS (0.7) & $8 / 33(24 \%)$ \\
\hline$+13 q 22$ & $1 / 18(6 \%)$ & NS (0.8) & $2 / 28(7 \%)$ & 0.01 & $11 / 33(33 \%)$ \\
\hline -15q15-qter & $1 / 18(6 \%)$ & NS $(0.4)$ & $4 / 28(14 \%)$ & NS (0.3) & $2 / 33(6 \%)$ \\
\hline$-17 p$ & $4 / 18(23 \%)$ & NS (0.7) & $5 / 28(18 \%)$ & NS (0.7) & $7 / 33(21 \%)$ \\
\hline$+18 q 21$ & $6 / 18(33 \%)$ & NS $(0.4)$ & $6 / 28(21 \%)$ & NS (0.8) & $8 / 33(24 \%)$ \\
\hline$+21 \mathrm{q}$ & $1 / 18(6 \%)$ & NS (0.4) & $4 / 28(14 \%)$ & NS (0.1) & $1 / 33(3 \%)$ \\
\hline$-22 q$ & $0 / 18(0 \%)$ & NS (0.4) & $1 / 28(4 \%)$ & NS (0.07) & $6 / 33(18 \%)$ \\
\hline $\begin{array}{l}\text { Mean number of } \\
\text { CGH alterations }\end{array}$ & 2.2 & 0.0001 & 5.4 & NS (0.6) & 5.1 \\
\hline
\end{tabular}

DLBCL, diffuse large B-cell lymphoma; FL, follicular lymphoma. Each CGH alteration in subsequent relapses from the same case was counted once, in tumors of the same diagnosis (FL/DLBCL). For mean number of CGH alterations, all tumors were included. Bold style indicates a statistically significant $P$-value, NS indicates a not statistically significant $P$-value.

Table 4 Most frequent CGH alterations in follicular lymphoma in relation to the total number of alterations

\begin{tabular}{|c|c|c|c|c|c|c|c|c|c|}
\hline Tumor no. & No. of alterations & $+1 q 31-32$ & $+X q 25-26$ & $+18 q 21$ & $+12 q 15$ & $-17 p$ & $+11 q 22$ & $+13 q 22$ & -9p23-pter \\
\hline 1 & 0 & & & & & & & & \\
\hline 2 & 0 & & & & & & & & \\
\hline $16: 1$ & 0 & & & & & & & & \\
\hline $18: 1$ & 0 & & & & & & & & \\
\hline $27: 1$ & 0 & & & & & & & & \\
\hline $30: 1$ & 0 & & & & & & & & \\
\hline $30: 2$ & 0 & & & & & & & & \\
\hline $21: 1$ & 1 & $+1 q$ & & & & & & & \\
\hline $23: 1$ & 1 & & & & & & & & \\
\hline $15: 1$ & 2 & & $+\mathrm{X}$ & & & & & & \\
\hline $17: 1$ & 2 & & $+\mathrm{X}$ & & & & & & \\
\hline $22: 1$ & 2 & & & $+18 q 21$ & $+12 q 13-15$ & & & & \\
\hline $30: 3$ & 2 & $+1 q$ & $+\mathrm{X}$ & & & & & & \\
\hline $30: 4$ & 2 & $+1 q$ & $+\mathrm{X}$ & & & & & & \\
\hline 19:1 & 3 & & & +18pter-q21 & $+12 q 11-21$ & $-17 p$ & & & \\
\hline $28: 1$ & 3 & & $+\mathrm{X}$ & & & & & & \\
\hline $24: 1$ & 3 & $+1 q 23-32$ & & +18 & & & & & \\
\hline $20: 1$ & 3 & & & & & & & & \\
\hline $25: 1$ & 4 & $+1 q$ & & $+18 q$ & & -17 & +11 & & \\
\hline $29: 2$ & 5 & & & +18 & & & & $+13 q 21-31$ & \\
\hline $29: 3$ & 5 & & & & & & & & \\
\hline $29: 1$ & 6 & & & +18 & & -17 & & $+13 q 21-31$ & \\
\hline $26: 1$ & 6 & & & +18 & & $-17 p$ & & & $-9 p$ \\
\hline
\end{tabular}

Bold style indicates a high-level amplification.

whereas $-22 q$ and $+3 q 24-25$ were identified as late events (Table 5).

Secondly, the CGH alterations were compared between individual tumors from the same patients in the 16 cases from which paired follicular lymphoma and transformed DLBCL were available (Figure 2 and Tables 2 and 6). From cases 27, 28, 29, and 30, three or more tumors were studied (Table 2). Based on the results, tentative schemes for the subsequent acquirement of CGH alterations were constructed (Figure 2). In addition, by comparing CGH aberrations in paired follicular lymphoma and transformed DLBCL from cases 15-26, the alterations + Xq25-26, -4q13-21, -6q16-21, and -8p22- 
Table 5 The most frequent CGH alterations in transformed diffuse large B-cell lymphoma in relation to the total number of alterations

\begin{tabular}{|c|c|c|c|c|c|c|c|c|c|c|c|c|c|c|c|}
\hline $\begin{array}{l}\text { Tumo } \\
\text { no. }\end{array}$ & $\begin{array}{l}\text { No. of } \\
\text { alterations }\end{array}$ & $-4 q 13-21$ & $+X q 25-26$ & -8p22-pter & -9p23-pter & $+7 p t e r-q 22$ & $+1 q 31-32$ & $+12 q 15$ & $+18 q 21$ & $-6 q 16-21$ & $+11 q 22$ & $-17 p$ & $+13 q 22$ & $-22 q$ & $+3 q 24-25$ \\
\hline 3 & 0 & & & & & & & & & & & & & & \\
\hline $23: 2$ & 1 & $-4 q$ & & & & & & & & & & & & & \\
\hline $24: 2$ & 2 & & +Xq21-qter & & & & & & & & & & & & \\
\hline 4 & 2 & & & -8p22-pter & -9p23-pter & & & & & & & & & & \\
\hline $21: 2$ & 2 & & & & & +7 & $+1 q$ & & & & & & & & \\
\hline $27: 2$ & 2 & & & & & & & & & & & & & & \\
\hline 7 & 3 & & & & & +7 & & & & & & & & & \\
\hline 6 & 3 & & & & & & & +12 pter-q22 & & & & & & & \\
\hline 5 & 3 & & $+\mathrm{Xp11-q26}$ & -8p22-pter & -9 p22-pter & & & & & & & & & & \\
\hline $20: 2$ & 3 & & & & & & & & & & & & & & \\
\hline $22: 2$ & 4 & & & $-8 p$ & & & & $+12 q 13-15$ & $+18 q 21$ & & & & & & \\
\hline 8 & 4 & $-4 q 10-22$ & & & & & & +12pter-q22 & $+18 q$ & $-6 q 14-21$ & & & & & \\
\hline $17: 2$ & 4 & & $+\mathrm{X}$ & & & & & $+12 q 10-21$ & & & & & & & \\
\hline $30: 5$ & 4 & & $+\mathrm{X}$ & & & & $+1 q$ & & & $-6 q$ & & & & & \\
\hline $19: 2$ & 4 & & & & & & & & +18pter-q21 & $-6 q 15-23$ & +11 & $-17 p$ & & & \\
\hline $25: 2$ & 4 & & $+\mathrm{X}$ & & & & & & $+18 q$ & & +11 & & & & \\
\hline $28: 2$ & 4 & & +Xq25-qter & & & +7 & & & & & & & & & \\
\hline $28: 3$ & 4 & & $+\mathrm{X}$ & & & +7 & & & & & & & & & \\
\hline $12: 2$ & 4 & & & & & & $+1 q$ & & & & & & & & \\
\hline $15: 2$ & 5 & & & & & & & & $+18 q 12-22$ & & $+11 q 14-22$ & & $+13 q 12-22$ & & \\
\hline $12: 1$ & 5 & & & & & & $+1 q$ & & & & & & & & \\
\hline $30: 6$ & 5 & & $+\mathrm{X}$ & & & & $+1 \mathrm{q}$ & +12 & & $-6 q$ & & & & & \\
\hline $27: 3$ & 5 & & $+\mathrm{X}$ & & & & & & +18pter-q21 & & & & & & \\
\hline 9 & 6 & & & & & & & & & -6q16-qter & $+11 q 13-22$ & & & & \\
\hline $26: 2$ & 7 & & & & $-9 p$ & & & & & & & $-17 p$ & & & \\
\hline 10 & 7 & $-4 q 13-21$ & & -8p12-pter & & & +1q31-qter & $+12 \mathrm{p} 11-\mathrm{q} 21$ & & & $+11 q 22-23$ & & & & \\
\hline $14: 1$ & 8 & & $+\mathrm{X}$ & & & +7 & & & & $-6 q$ & & $-17 p$ & & & \\
\hline $16: 2$ & 8 & -4q21-qter & $+X q$ & $-8 p$ & & & & & & & & $-17 p$ & & & \\
\hline $18: 1$ & 9 & & & & & & & $+12 q 15-22$ & & & & & & & \\
\hline 11 & 9 & & & & & +7pter-q22 & & & & & & & & & \\
\hline $13: 2$ & 10 & & $+\mathrm{X}$ & & & +7 & & +12p12-q21 & & $-6 \mathrm{p} 21-\mathrm{q} 21$ & & & & $-22 q$ & \\
\hline $13: 1$ & 11 & -4 pter-q21 & & & & +7 & & $+12 \mathrm{p} 12-\mathrm{q} 21$ & & $-6 \mathrm{p} 21-\mathrm{q} 21$ & & & & & \\
\hline $29: 4$ & 12 & & & & & +7pter-q31 & & & & $-6 q 14-25$ & & $-17 p$ & $+13 q 22$-qter & & +3q25-qter \\
\hline $14: 2$ & 12 & & $+\mathrm{X}$ & & & +7 & & & & $-6 q$ & & $-17^{1}$ & & & \\
\hline $14: 3$ & 12 & & $+\mathrm{X}$ & & & +7 & & & & $-6 q$ & & -17 & & & \\
\hline
\end{tabular}

Bold style indicates a high-level amplification. 
Table 6 Acquired alterations in transformed DLBCL from the 16 studied cases with matched follicular lymphoma and DLBCL tumors

\begin{tabular}{|c|c|c|c|}
\hline \multirow[t]{2}{*}{ Case } & \multicolumn{3}{|c|}{$\begin{array}{c}\text { Acquired CGH alterations in DLBCL (total number } \\
\text { and regions involved) }\end{array}$} \\
\hline & $>2^{\mathrm{a}}$ (frequent) & $2^{\mathrm{a}}$ (less frequent) & $<2^{\text {a }}($ rare $)$ \\
\hline 15 & & $\begin{array}{l}+4 q 31-q \text { ter } \\
+11 q 14-22\end{array}$ & $\begin{array}{l}13 q 12-22 \\
18 q 12-22 \\
-9 q 22\end{array}$ \\
\hline 16 & $\begin{array}{l}+X q \\
+8 q 21-q t e r \\
\text {-4q21-qter }\end{array}$ & $\begin{array}{l}-1 \mathrm{p} 32-\text {-pter } \\
-8 \mathrm{p} \\
-17 \mathrm{p}\end{array}$ & $\begin{array}{l}+9 \mathrm{q} 22 \text {-qter } \\
-15\end{array}$ \\
\hline 17 & & $+12 q 10-21$ & $-16 q 21-23$ \\
\hline 18 & $+8 q 12-24$ & $\begin{array}{l}+4 q \\
+12 q 15-22\end{array}$ & $\begin{array}{l}+2 q 22-33 \\
+6 q 10-21 \\
+9 p \\
+12 p 12 \\
-6 p 21 \\
-11 q 12-13\end{array}$ \\
\hline 19 & $-6 q 15-23$ & +11 & \\
\hline 20 & & & \\
\hline 21 & +7 & & \\
\hline 22 & & $\begin{array}{l}-8 \mathrm{p} \\
-19 \mathrm{pter}-13.2\end{array}$ & \\
\hline 23 & $-4 q$ & & \\
\hline 24 & + Xq21-qter & $+11 p$ & \\
\hline 25 & $+\mathrm{X}$ & & \\
\hline 26 & & $\begin{array}{l}-1 p 21-32 \\
-19 p\end{array}$ & \\
\hline 27 & $+8 q 21.3$-qter & & $+8 p$ \\
\hline 28 & +7 & & $+2 \mathrm{p} 14-16$ \\
\hline 29 & $\begin{array}{l}+7 \text { pter-q31 } \\
+8 q 10-22 \\
-4 q 32-33 \\
-6 q 14-25\end{array}$ & $-17 p$ & $\begin{array}{l}+3 q 25 \text {-qter } \\
+10 p \\
-13 q 22-q t e r \\
-X\end{array}$ \\
\hline 30 & $-6 q$ & & $+17 q$ \\
\hline
\end{tabular}

DLBCL, diffuse large B-cell lymphoma.

${ }^{\mathrm{a}}>2,2$, and $<2$ indicate the no. of cases in which each specified alteration was observed. Each alteration in subsequent DLBCL relapses from the same case was counted once.

pter were repeatedly found in transformed DLBCL only (three or more cases) but never in the follicular lymphoma (Table 6).

\section{Clonality}

The results from the CGH analyses indicated that the tumors from the 16 matched cases were clonally related. However, in cases 27 and 29, the clonal relation remains unclear after CGH analysis as well as the mutual order of the subsequent tumors, respectively. Tumor 27:2 was shown to display two $V_{\mathrm{H}}$ rearrangements, that is, one $V_{\mathrm{H}} 3$ and one $V_{\mathrm{H}} 5$ rearrangement (Figure 2) whereas tumors 27:1 and 27:3 present $V_{\mathrm{H}} 5$ and $V_{\mathrm{H}} 3$, respectively. This indicates that there is a clonal relation between all three tumors and a clonal heterogeneity, especially evident in tumor 27:2. For case 29, all four tumors showed a clonally related IGH rearrangement; however, tumors 29:1 as well as 29:2 shows unique mutations whereas $29: 3$ and $29: 4$ are $100 \%$ identical. Tumors 20:1, 20:2, 22:1, and 22:2 were used as control as the tumors from respective case showed overlapping CGH profiles indicating clonality. The tumors from respective cases showed identical $I G H$ rearrangements thus clonally related (not shown).

\section{CGH Alterations in Relation to Clinical Parameters}

The clinical course of the 30 included patients was poor. At the initial lymphoma diagnosis, four patients presented a discordant lymphoma with a simultaneous diagnosis of follicular lymphoma and transformed DLBCL in different sites. In the other 26 patients, the time interval between initial diagnosis of follicular lymphoma and subsequently of transformed DLBCL varied from 0.5 to 247 months (Table 1). However, no differences in CGH alterations were observed between cases with short (below median) or long (above median) time interval from initial follicular lymphoma diagnosis to transformation (Table 1). At the end of follow-up, only three patients were alive and disease-free, two of whom were treated with high-dose chemotherapy and autologous stem cell transplantation. The total number of CGH alterations in these tumors varied from 0 to 12, without predominant involvement of a specific chromosomal region.

\section{Distinct CGH Alterations in Transformed as Compared to De Novo DLBCL}

The CGH alterations found in transformed DLBCL were compared to our previously published data for de novo DLBCL (Table 3). This revealed that losses involving $-4 q 13-21$ were significantly more common in transformed DLBCL as compared to de novo DLBCL $(P=0.02$; Table 3$)$. In contrast, $+3 q 24-25$ and $+13 q 22$ were significantly more frequent in $d e$ novo DLBCL than transformed DLBCL $(P=0.04$ and 0.01 , respectively; Table 3 ). No other differences were detected and the total numbers of CGH aberrations were similar in the two groups (5.1 vs $5.4 ; P=0.6)$.

\section{Discussion}

In approaching the molecular mechanism leading to transformation from indolent follicular lymphoma 

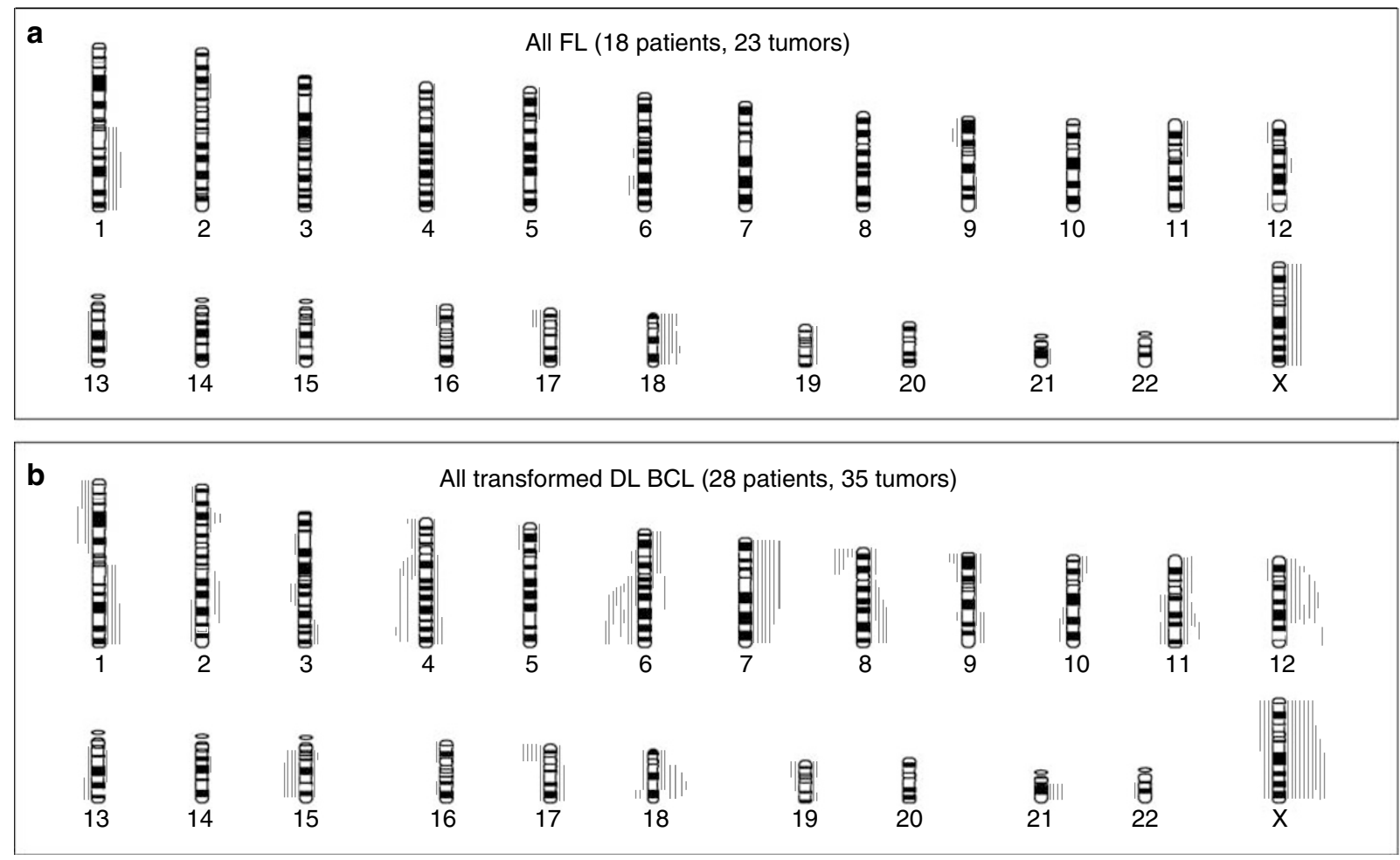

Figure 1 Copy number changes detected by CGH in 23 follicular lymphomas from 18 patients (a) and 35 transformed DLBCLs from 28 patients (b). Each line represents one alteration detected in one tumor, with losses indicated to the left and gains to the right of the ideograms. Thick lines indicate high-level amplifications. In cases with more than one sample analyzed of the same diagnosis (follicular lymphoma or transformed DLBCL), each alteration was counted once.

to aggressive DLBCL, we have comprehensively characterized genomic copy number imbalances in a panel of 58 such tumors from a cohort of 30 patients diagnosed with transformed DLBCL following or in parallel with an initial diagnosis of follicular lymphoma. Based on the results, we conclude that transformation to DLBCL is associated with increasing numbers of acquired CGH alterations, with predominantly involving non-random chromosomal regions. The findings add to the present knowledge of molecular genetic events associated with the transformation of follicular lymphoma that involves both mutations and deregulation of several cancer-related genes as well as acquired structural and numerical alterations of multiple chromosomal regions.

The patterns of CGH alterations detected in follicular lymphoma and transformed DLBCL suggested a progression of genetic events. These results are in agreement with previous studies concerning clonal evolution in follicular lymphoma, ${ }^{2,22}$ suggesting that secondary mutations accumulate and the sum of the different genes involved produces the transformation. For this purpose, the detected alterations were related to the total number of CGH alterations and their relation to clinically evident transformed DLBCL. The data underlying these evaluations are illustrated in Tables 4-6 and Figure 2. The alterations -4q13-21, -8p22-pter,
+ Xq25-26, + 1q31-32, and + 7pter-q22 appeared as early events in the transformed DLBCL (Table 5), whereas + Xq25-26, +1q31-32, +12q15, and + 18q21 appeared early in follicular lymphoma tumors (Table 4). Figure 2 illustrates the acquired CGH alterations in paired samples of follicular lymphoma and transformed DLBCL analyzed from the same patients. The model we constructed from the results above (Figure 3) is in close agreement with previous studies concerning regions involved in the transformation. ${ }^{5,23}$ As previously noted in other reports, gains tended to be more common in early follicular lymphoma whereas losses appeared in late follicular lymphoma tumor progression. ${ }^{22}$ It can be speculated that late progression/transformation in follicular lymphoma depends on alterations in rate-limiting genes, that is, tumor suppressor genes rather than oncogenes. Alterations of some chromosomes were detected as early events in both follicular lymphoma and transformed DLBCL, possibly indicating that genes in these regions, for example on chromosome $\mathrm{X}$, may be involved in the tumor progression as well as in tumor initiation. Some transitional changes, that is CGH alterations found only in the first available DLBCL and not in any of the follicular lymphoma from the same patients, were recurrently detected in our series (Table 6). Interestingly, -6q16-21 and + 7pter-q22 were both detected as transitional changes in three different cases, further 

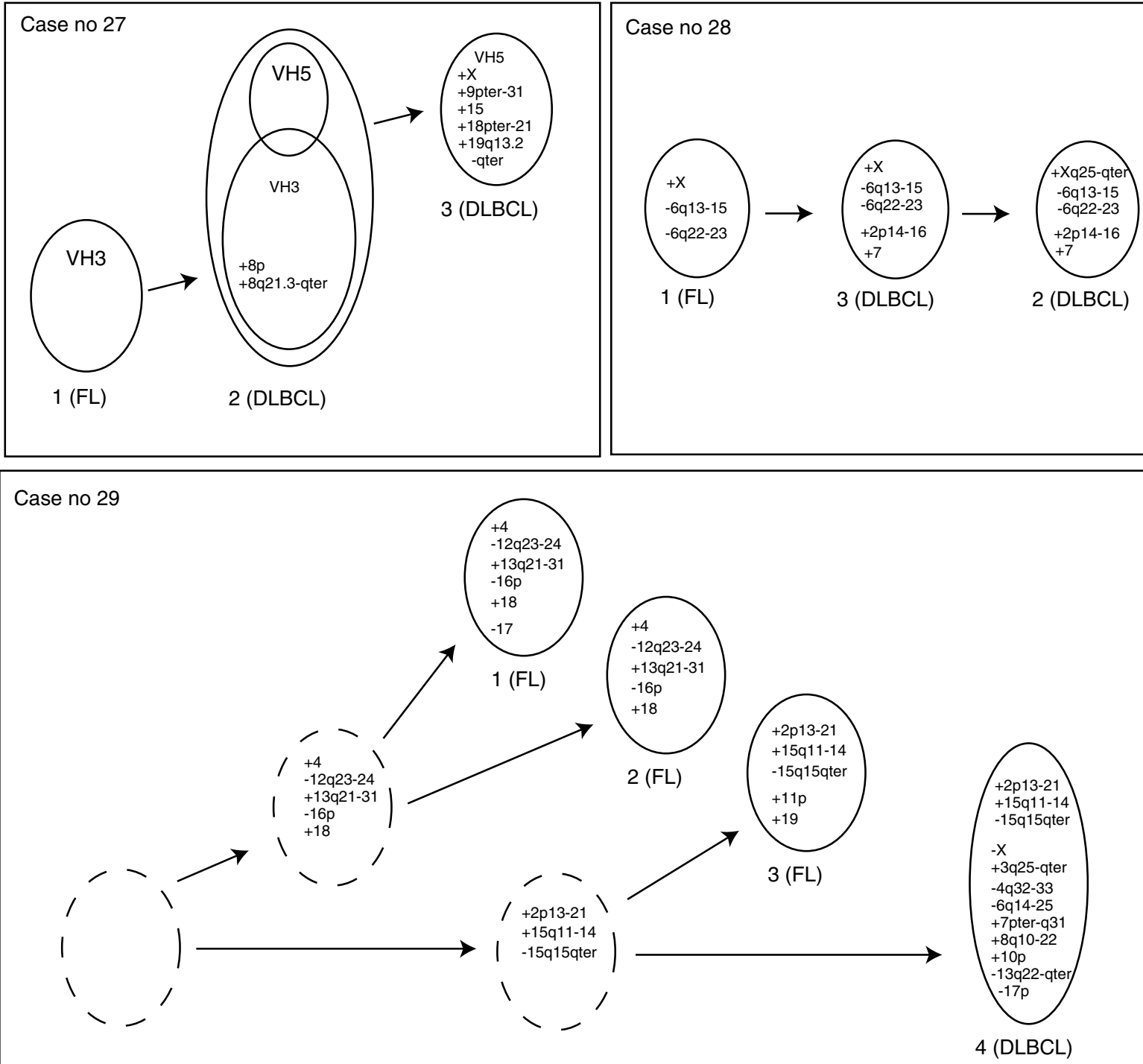

Case no 30

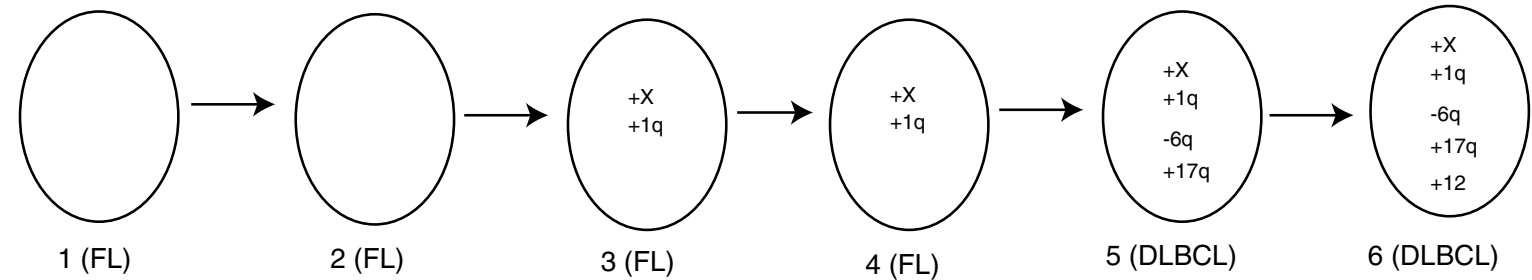

Figure 2 Schematic diagram illustrating CGH alterations acquired during progression from follicular lymphoma to transformed DLBCL in cases 27-30. Observe that the order of genetic progression indicated from left to right is not necessarily same as the chronological order. 


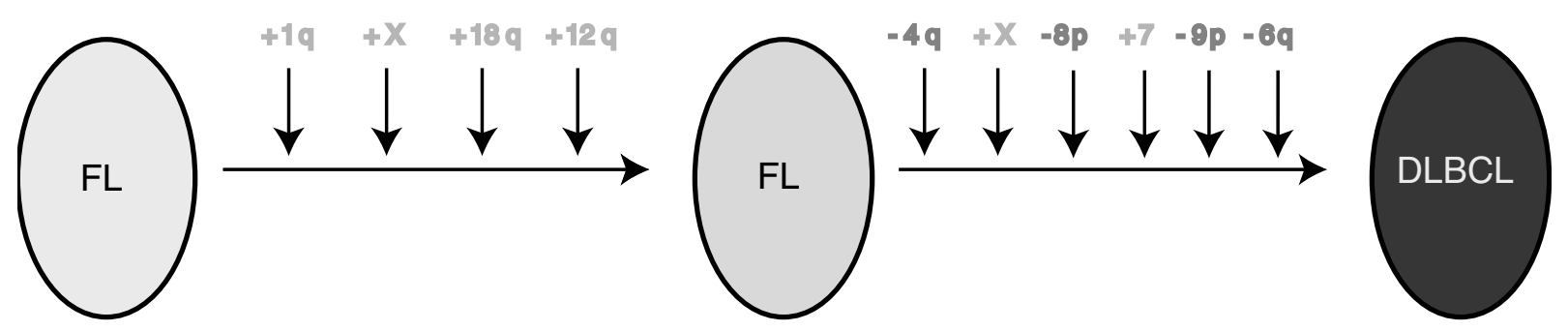

Increasing total number of cytogenetic alterations

Figure 3 A hypothetical model for tumor progression and transformation based on changes detected by CGH. The model is based on the combined results from the diagnostic follicular lymphoma tumor, the transformed DLBCLs, and relapses studied. A plus $(+)$ indicates chromosomal gains, and a minus (-) denotes losses. The indicated chromosomal arms refer to 1q31-32, 18q21, 12q15, respectively.

Table 7 Alterations reported as specific for transformed diffuse large B-cell lymphoma

\begin{tabular}{|c|c|c|c|c|c|c|}
\hline Alteration & Hough et $\mathrm{al}^{16}$ & Goff et $\mathrm{al}^{8}$ & Nagy et $\mathrm{al}^{24}$ & Boonstra et $\mathrm{al}^{13}$ & Present study & Martinez-Climent et al ${ }^{12}$ \\
\hline$+\mathrm{Xq} 25-26$ & $2(9 \%)$ & $0(0 \%)$ & $0(0 \%)$ & $2(33 \%)$ & $4(25 \%)$ & $2(20 \%)$ \\
\hline$+3 q 24-25$ & $1(4 \%)$ & $0(0 \%)$ & $0(0 \%)$ & $0(0 \%)$ & $1(6 \%)$ & $1(10 \%)$ \\
\hline$-4 q 21-q t e r$ & $0(0 \%)$ & $1(17 \%)$ & $0(0 \%)$ & $1(17 \%)$ & $3(19 \%)$ & $2(20 \%)$ \\
\hline$-6 q 16-21$ & $0(0 \%)$ & $0(0 \%)$ & $2(40 \%)$ & $2(33 \%)$ & $3(19 \%)$ & $1(10 \%)$ \\
\hline +7pter-q22 & $2(9 \%)$ & $1(17 \%)$ & $0(0 \%)$ & $5(83 \%)$ & $3(19 \%)$ & $1(10 \%)$ \\
\hline -8p22-pter & $2(9 \%)$ & $1(17 \%)$ & $0(0 \%)$ & $0(0 \%)$ & $2(12 \%)$ & $0(0 \%)$ \\
\hline -9p23-pter & $0(0 \%)$ & $0(0 \%)$ & $1(20 \%)$ & $1(17 \%)$ & $1(6 \%)$ & $0(0 \%)$ \\
\hline$+11 q 22$ & $0(0 \%)$ & $0(0 \%)$ & $0(0 \%)$ & $1(17 \%)$ & $2(12 \%)$ & $0(0 \%)$ \\
\hline$+12 q 15$ & $1(4 \%)$ & $1(17 \%)$ & $0(0 \%)$ & $1(17 \%)$ & $3(19 \%)$ & $0(0 \%)$ \\
\hline$+13 q 22$ & $0(0 \%)$ & $1(17 \%)$ & $0(0 \%)$ & $0(0 \%)$ & $1(6 \%)$ & $0(0 \%)$ \\
\hline$-17 p$ & $0(0 \%)$ & $1(17 \%)$ & $0(0 \%)$ & $2(33 \%)$ & $1(6 \%)$ & $1(10 \%)$ \\
\hline$+18 q 21$ & $1(4 \%)$ & $2(33 \%)$ & $2(40 \%)$ & $1(17 \%)$ & $2(12 \%)$ & $2(20 \%)$ \\
\hline Cases studied & 23 & 6 & 5 & 6 & 16 & 10 \\
\hline Method & $\mathrm{CGH}$ & $\mathrm{CGH}$ & CGH & CGH & CGH & Array-CGH \\
\hline
\end{tabular}

CGH alterations are detailed which were found in transformed diffuse large B-cell lymphoma only, but were absent in matching FLs from the same patient.

supporting their relevance for the transformation process.

A linear clonal evolution of events was suggested for three of the four cases from which three or more consecutive tumors were studied. However, case no 27 illustrates a more complicated picture (Figure 2) showing that the diagnostic tumor (27:1) and the second relapse (27:3) are not closely related. A clonal heterogeneity was found in the first relapse (27:2), showing a clonal relation to tumor $27: 1$ as well as 27:3. Notably, this patient transformed to DLBCL only 3 months after the initial follicular lymphoma diagnosis and it can be suggested that this heterogeneity within the tumor facilitates the transformation process from follicular lymphoma to DLBCL. It has been shown that clonal evolution in follicular lymphoma to transformed DLBCL in most cases is linear ${ }^{13}$ but alternatively, and more complicated, non-linear progression where the tumor originates from an earlier stage of tumor differentiation is also common in follicular lymphoma as well as in follicular lymphoma to DLBCL transformation.
This scenario is evident for case 29 (Figure 2), where all four tumors are clonally related even if the tumor progression is not linear. Two lineages were detected (Figure 2).

Transformed DLBCL were found to harbor significantly more genomic copy number imbalances than the corresponding follicular lymphoma $(P=0.0001)$, which is in agreement with previous reports. ${ }^{12,16,24}$ Karyotypic analysis has also shown that transformed DLBCL are cytogenetically more unstable, resulting in a scattered picture of numerical alterations. ${ }^{17}$ In close agreement with previous studies, ${ }^{5,12,13}$ the most common alterations in our series of transformed DLBCL were + Xq21-26 (36\%), +7 pter-q22 (25\%), +12q15 (29\%), and + $18 \mathrm{q} 21$ $(21 \%)$, as well as $-6 q 16-21(25 \%),-8 p 22$-pter $(18 \%)$, and $-17 \mathrm{p}(18 \%)$.

Genomic copy number alterations associated with transformed DLBCL have been previously reported in five different studies applying conventional CGH or array-CGH (Table 7). Our findings of frequent Xq25-26, + 7pter-q22, + 12q15, + 18q21, -4q21-qter, 
-6q16-21, and $-17 p$ were also demonstrated in three or more of the previous reports (Table 7). We found that two of these alterations, -6q16-21 and +7 pter-q22, were significantly more frequent in transformed DLBCL than in follicular lymphoma (Table 3). Losses in $6 q$ and gains of $7 q$ has been described before in follicular lymphoma tumors, ${ }^{25}$ in DLBCL tumors transformed from follicular lymphoma, ${ }^{26}$ as well as in de novo DLBCL. ${ }^{17,27}$ Interestingly, a minimal region of deletion at 6q16.3 in follicular lymphoma has recently been described, ${ }^{25}$ that includes the candidate gene $R N A H$, encoding a RNA helicase that can bind the $p 16^{I N K 4 a}$ promoter and positively regulates its transcription. Loss of $p 16^{I N K 4 a}$ has been suggested to be involved in the transformation from follicular lymphoma to DLBCL. ${ }^{5}$ Concerning chromosome 7 , two candidate genes located at 7q11.2-22, that is, CYP51 and CUTL1, have been found overexpressed in lymphomas. ${ }^{12}$ CUTL1 is particularly interesting as it regulates lymphopoiesis and is associated with lymphoid malignancies in mice ${ }^{28}$ Concerning the alterations $-17 p$ and $+12 q 15$, possible candidate target genes is TP53 in $17 \mathrm{p} 13.1$ and MDM2 in 12q14.3-15. Previous reports of transformed DLBCL have documented alterations of MDM2, as well as a limited role of TP53 mutations. ${ }^{23,29}$ In our study, the chromosomal loci for TP53 and MDM2 were involved in $46 \%$ of all cases; however, they were never observed in the same patient. This indicates that deregulation of p53 expression or downstream inhibition is one of the main reasons for the transformation and a possible future therapeutic target.

The patterns of CGH alterations found in transformed DLBCL was partly overlapping and partly distinct from our previous findings in de novo DLBCL. ${ }^{17}$ The alterations $-6 q 16-21$ and +7 pter-q22 that distinguished transformed DLBCL from follicular lymphoma were also frequently found in de novo DLBCL. This suggests that de novo and transformed DLBCL involve overlapping etiological mechanisms. In contrast, overt differences between the groups included significant over-representation of +3q2425 and +13q22 and under-representation of -4q1321 in de novo DLBCL as compared to transformed DLBCL. Clinically, the recognition of transformed DLBCL is important, as this entity is associated with a more adverse outcome than the de novo cases. Therefore, the findings of distinguishing genetic alterations could potentially be utilized for diagnostic purposes to distinguish between de novo and transformed DLBCL.

Clinical progression from follicular lymphoma to transformed DLBCL is on the genetic level associated with acquirement of increasing number of genomic copy number changes and non-random involvement of specific target regions. It is expected that precise identification of the genes targeted by losses in 6q and gains in 7 will improve our understanding of the transition from follicular lymphoma to DLBCL.
Taken together, the available information concerning molecular genetic alterations in these tumor groups suggests that multiple events are necessary to achieve transformation. Therefore, an important task is to delineate the sequential order of these events and their possible influence on the clinical outcome. These may in turn reflect pathways and events that could be utilized for prognostic and/or therapeutic purposes. $^{22}$

\section{Acknowledgements}

This study was financially supported by the Swedish Cancer Society, the Magn. Bergwall Foundation, the Swedish Medical Association, the Swedish Research Council, and the Stockholm County Council.

\section{References}

1 Nathwani BN, Harris NL, Weisenburger D, et al. Follicular Lymphoma. In: Jaffe ES, Harris NL, Stein H, Vardiman JW (eds). Tumors of Haematopoetic and Lymphoid Tissues. International Agency for Research on Cancer (IARC) Press: Lyon, France, 2001, pp 162-167.

2 Viardot A, Barth TF, Moller P, et al. Cytogenetic evolution of follicular lymphoma. Semin Cancer Biol 2003;13:183-190.

3 Bakhshi A, Jensen JP, Goldman P, et al. Cloning the chromosomal breakpoint of $\mathrm{t}(14 ; 18)$ human lymphomas: clustering around $\mathrm{JH}$ on chromosome 14 and near a transcriptional unit on 18. Cell 1985;41:899-906.

4 Weiss LM, Warnke RA, Sklar J, et al. Molecular analysis of the $t(14 ; 18)$ chromosomal translocation in malignant lymphomas. N Engl J Med 1987;317:11851189.

5 Lossos IS, Levy R. Higher grade transformation of follicular lymphoma: phenotypic tumor progression associated with diverse genetic lesions. Semin Cancer Biol 2003;13:191-202.

6 Sander CA, Yano T, Clark HM, et al. p53 mutation is associated with progression in follicular lymphomas. Blood 1993;82:1994-2004.

7 Yano T, Jaffe ES, Longo DL, et al. MYC rearrangements in histologically progressed follicular lymphomas. Blood 1992;80:758-767.

8 Goff LK, Neat MJ, Crawley CR, et al. The use of realtime quantitative polymerase chain reaction and comparative genomic hybridization to identify amplification of the REL gene in follicular lymphoma. Br J Haematol 2000;111:618-625.

9 Matolcsy A, Casali P, Warnke RA, et al. Morphologic transformation of follicular lymphoma is associated with somatic mutation of the translocated Bcl-2 gene. Blood 1996;88:3937-3944.

10 Elenitoba-Johnson KS, Gascoyne RD, Lim MS, et al. Homozygous deletions at chromosome 9p21 involving p16 and p15 are associated with histologic progression in follicle center lymphoma. Blood 1998;91:46774685 .

11 King BE, Chen C, Locker J, et al. Immunophenotypic and genotypic markers of follicular center cell neo- 
plasia in diffuse large B-cell lymphomas. Mod Pathol 2000;13:1219-1231.

12 Martinez-Climent JA, Alizadeh AA, Segraves R, et al. Transformation of follicular lymphoma to diffuse large cell lymphoma is associated with a heterogeneous set of DNA copy number and gene expression alterations. Blood 2003;101:3109-3117.

13 Boonstra R, Bosga-Bouwer A, Mastik M, et al. Identification of chromosomal copy number changes associated with transformation of follicular lymphoma to diffuse large B-cell lymphoma. Hum Pathol 2003; 34:915-923.

14 de Vos S, Hofmann WK, Grogan TM, et al. Gene expression profile of serial samples of transformed B-cell lymphomas. Lab Invest 2003;83:271-285.

15 Lossos IS, Alizadeh AA, Diehn M, et al. Transformation of follicular lymphoma to diffuse large-cell lymphoma: alternative patterns with increased or decreased expression of c-myc and its regulated genes. Proc Natl Acad Sci USA 2002;99:8886-8891.

16 Hough RE, Goepel JR, Alcock HE, et al. Copy number gain at 12q12-14 may be important in the transformation from follicular lymphoma to diffuse large B cell lymphoma. Br J Cancer 2001;84:499-503.

17 Berglund M, Enblad G, Flordal E, et al. Chromosomal imbalances in diffuse large B-cell lymphoma detected by comparative genomic hybridization. Mod Pathol 2002;15:807-816.

18 Jaffe ES, Harris NL, Stein $\mathrm{H}$, et al. Pathology and Genetics of Tumours of Haematopoietic and Lymphoid Tissues. IARC Press: Lyon, 2001.

$19 \mathrm{Li} \mathrm{AH}$, Rosenquist R, Forestier E, et al. Clonal rearrangements in childhood and adult precursor $\mathrm{B}$ acute lymphoblastic leukemia: a comparative polymerase chain reaction study using multiple sets of primers. Eur J Haematol 1999;63:211-218.

$20 \mathrm{Li} \mathrm{AH,} \mathrm{Rosenquist} \mathrm{R,} \mathrm{Forestier} \mathrm{E,} \mathrm{et} \mathrm{al.} \mathrm{Detailed}$ clonality analysis of relapsing precursor $\mathrm{B}$ acute lymphoblastic leukemia: implications for minimal residual disease detection. Leukemia Res 2001;25: 1033-1045.

21 Thorselius M, Walsh S, Eriksson I, et al. Somatic hypermutation and $\mathrm{V}(\mathrm{H})$ gene usage in mantle cell lymphoma. Eur J Haematol 2002;68:217-224.

22 Hoglund M, Sehn L, Connors JM, et al. Identification of cytogenetic subgroups and karyotypic pathways of clonal evolution in follicular lymphomas. Genes Chromosomes Cancer 2004;39:195-204.

23 Sigal S, Ninette A, Rechavi G. Microarray studies of prognostic stratification and transformation of follicular lymphomas. Best Pract Res Clin Haematol 2005; 18:143-156.

24 Nagy M, Balazs M, Adam Z, et al. Genetic instability is associated with histological transformation of follicle center lymphoma. Leukemia 2000;14:2142-2148.

25 Henderson LJ, Okamoto I, Lestou VS, et al. Delineation of a minimal region of deletion at 6q16.3 in follicular lymphoma and construction of a bacterial artificial chromosome contig spanning a 6-megabase region of 6q16-q21. Genes Chromosomes Cancer 2004;40:60-65.

26 Richardson ME, Chen QG, Filippa DA, et al. Intermediate- to high-grade histology of lymphomas carrying $\mathrm{t}(14 ; 18)$ is associated with additional nonrandom chromosome changes. Blood 1987;70:444-447.

27 Monni O, Joensuu H, Franssila K, et al. DNA copy number changes in diffuse large B-cell lymphomacomparative genomic hybridization study. Blood 1996;87:5269-5278.

28 Sinclair AM, Lee JA, Goldstein A, et al. Lymphoid apoptosis and myeloid hyperplasia in CCAAT displacement protein mutant mice. Blood 2001;98:36583667.

29 Davies AJ, Lee AM, Taylor C, et al. A limited role for TP53 mutation in the transformation of follicular lymphoma to diffuse large B-cell lymphoma. Leukemia 2005;19:1459-1465. 